\title{
Original
}

\section{Development of ITER TF Coil Assembly Technique, Integration of Winding Pack into Coil Case}

\author{
Mio NAKAMOTO ${ }^{* 1, \dagger}$, Yuma KASAI ${ }^{* 1}$, Takashi BABA ${ }^{* 1}$, Kazuyuki SAKAMOTO ${ }^{* 1}$, \\ Tatsuya SHIMIZU ${ }^{* 1}$, Kengo SAITO ${ }^{* 1}$, Norikiyo KOIZUMI ${ }^{* 1}$, Masataka NAKAHIRA ${ }^{* 1}$, \\ Eikou FUJIWARA ${ }^{* 2}$, Minoru YAMANE ${ }^{* 3}$, Tsuneaki MINATO ${ }^{* 3}$ and Kazuo KUNO*3
}

\begin{abstract}
Synopsis: The ITER Toroidal Field (TF) coil is a D-shaped superconducting magnet. A set of 18 TF coils forms a donut shape when assembled around the ITER vacuum vessel. The magnetic property of a coil is characterized by a current center line (CCL). To serve their function as plasma containment magnets, severe requirement of $\varphi 2.6 \mathrm{~mm}$ cylindrical tolerance is defined for the critical portion of the TF coils. In previous study, the manufacturing tooling and procedure have been developed and applied for manufacturing of Winding Packs (WP) and TF Coil Case (TFCC) subassemblies. In integration of a WP into a TFCC, predetermined CCL of the WP shall be controlled and transferred to reference points of the TFCC. For precise control of the CCL positions, deformations of the WP and the TFCC must be controlled. Also, the precise tracking of the CCL position required some techniques to evaluate the CCL positions even after the WP is completely covered by the TFCC. Techniques have been developed through welding trials and structural simulation analysis. Those techniques are applied to TF coil production and two TF coils have been completed successfully.
\end{abstract}

Keywords: ITER, TF coil, WP insertion, closure welding, current center line (CCL)

(Some figures in this article may appear in color only in the electronic version)

\section{Introduction}

The ITER Toroidal Field (TF) coil is a D-shaped superconducting magnet. When $18 \mathrm{TF}$ coils are positioned around the vacuum vessel at 20 -degree interval, it forms a $3 \mathrm{D}$ donut shape. A function of TF coils is to generate a uniform magnetic field inside the ITER Vacuum Vessel to stably contain plasma in the region of interest. They are designed to generate maximum of $11.8 \mathrm{~T}$ magnetic field when a current of $68 \mathrm{kA}$ flows through the $\mathrm{Nb}_{3} \mathrm{Sn}$ conductors at $4.5 \mathrm{~K}$. For the uniformity, all of $18 \mathrm{TF}$ coils shall have desired common magnetic property to provide $5.3 \mathrm{~T}$ steady field in $6.2 \mathrm{~m}$ radius. The magnetic property of a coil can be characterized by a current center line $(\mathrm{CCL})^{1)}$. A tight tolerance of $\varphi 2.6 \mathrm{~mm}$ cylindrical tolerance is given to the CCL positions at straight portion

Received October 13, 2020

The views and opinions expressed herein do not necessarily reflect those of the ITER Organization.

*1 National Institutes for Quantum and Radiological Science and Technology, 801-1 Mukoyama, Naka, Ibaraki 311-0193, Japan

*2 Mitsubishi Heavy Industries, Ltd., 1-1 Wadasaki-cho 1-chome, Hyogo-ku, Kobe, Hyogo 652-8585, Japan

*3 Mitsubishi Electric Corporation Energy Systems Center, 1-1-2 Wadasaki-cho, Hyogo-ku, Kobe, Hyogo 652-8555, Japan

† E-mail: nakamoto.mio@qst.go.jp

DOI: $10.2221 /$ jcsj. 55.400 of the final coil.

Each of TF coils is composed of a conductor winding, called a Winding Pack (WP) and TF Coil Case (TFCC) structures $^{2)}$. A WP is composed of 7 Double Pancakes (DPs) stacked with precise alignment ${ }^{3)}$. There is severe tolerance of $\varphi 2 \mathrm{~mm}$ on CCL for the straight portion of WPs and the way to achieve the requirements have been developed $^{3)}$. A TFCC is divided into 4 main structures made of stainless steel, $\mathrm{AU}, \mathrm{BU}, \mathrm{AP}$ and $\mathrm{BP}^{2)}$.

The WP integration is performed by aligning a WP to the TFCC reference in consideration of CCL positions. The procedure and tooling for precise alignment during WP Insertion has been developed ${ }^{4)}$. However, a WP integration into a TFCC involved welding of the TFCC components together, the welding deformation has an impact on the final TF coil shape. Moreover, each WP is about $9 \mathrm{~m}$ by $14 \mathrm{~m}$ structure weighing 110 tons and it deforms by gravitational effect differently from the TFCC. Since the final CCL positions are defined with respect to the final TF coil shape, welding deformation and gravitational deformation must be controlled to achieve the tight tolerance requirement given to the $\mathrm{TF}$ coils.

Finally, the CCL positions determined for a WP shall 
be transferred to a TFCC. Since the WP is enclosed by the TFCC and cannot be measured directly after welding of the TFCC, a technique must be developed to evaluate the CCL positions through the WP integration process.

In this paper, high precision CCL control and evaluation techniques developed for the WP integration are described. With those techniques, the CCL positions determined for a WP are controlled and transferred to each TFCC regardless of the welding deformation and the gravitational deformation. Those techniques have been applied to all TF coils and two of them have been successfully completed. The manufacturing results of those two coils are also reported.

\section{WP Integration}

WP integration is performed in stepwise process. The targets for each step are determined to achieve the requirements in WP integration. To understand the technical issues in WP and TFCC integration, the manufacturing steps and the requirements are described and then, technical issues are explained below.

\subsection{Manufacturing steps}

As described in Fig. 1 of [5], each WP is integrated into a TFCC by following steps: insertion of a WP into AU and BU (WP Insertion), welding of the TFCC to enclose the WP (Closure Welding), filling of gaps between the WP and the TFCC (Gap-filling), machining of peripheral interfaces of the coil (Final Machining), and quality inspections of final coil (Factory Acceptance Tests).

\subsection{Requirements}

In WP integration, there are four major requirements; 1) high precision control of CCL positions, 2) tight tolerances on inter-coil and peripheral interfaces, 3) minimum wall thickness of TFCC for structural strength, and 4) minimum gap assurance between a WP and a TFCC for resin injection in Gap-filling.

The first requirement is to satisfy the tolerances defined

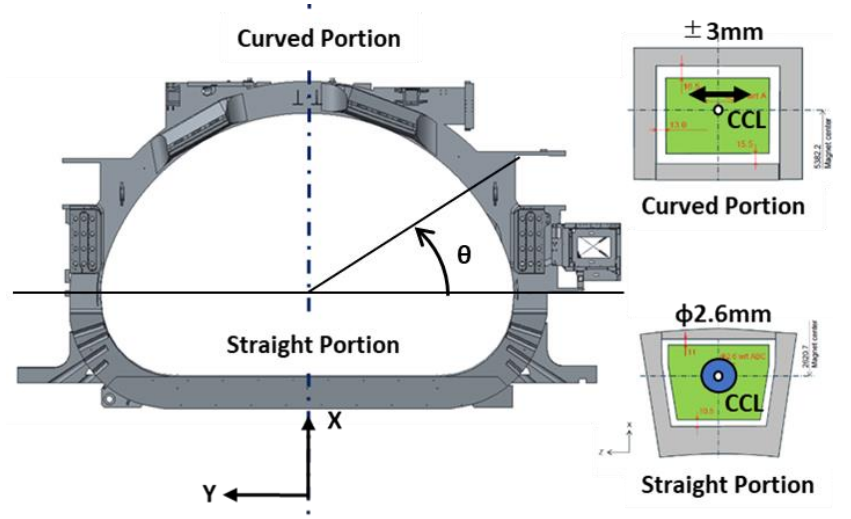

Fig. 1 CCL tolerances on final coils. on CCL positions of a TF coil. Since the straight portion of $\mathrm{TF}$ coils has more influence on the magnetic field generated by the coil, severe tolerance of $\varphi 2.6 \mathrm{~mm}$ is defined for the CCL positions at the straight portion while the requirements for curved portion is $\pm 3 \mathrm{~mm}$ (Fig. 1).

The second requirement defines the outer envelope of TF coil with respect to the CCL positions. The most severe tolerance of $\pm 0.2 \mathrm{~mm}$ is given at the profile of side surfaces of straight portions, inter-coil interfaces with neighboring TF coils.

The third requirement is satisfied during TFCC subassembly fabrication. This requirement defines the inner surface of the TFCC.

Then, the fourth requirement of minimum $4 \mathrm{~mm}$ gap limits the space for a WP within TFCC. For a WP, cylindrical tolerances of $\varphi 2 \mathrm{~mm}$ and $\varphi 4 \mathrm{~mm}$ are defined for CCL positions at the straight portion and curved portion, respectively ${ }^{3)}$. Due to possible non-uniformity in insulation thickness of a WP, the CCL positions of the WP do not necessarily coincide with the profile center of the WP.

As a result, the realization of the first requirement becomes very challenging. To satisfy the severe requirement, WP position shall be well controlled to minimize CCL deviations.

\subsection{Factors for deviations}

In WP integration, the straight portion of a WP and a TFCC are aligned first since the requirement on the straight portion is more severe. Then, the curved portion of the WP and the TFCC are adjusted. For precise alignment, it is important to minimize the impacts of deformations. The main factor of deformations for a TFCC is welding deformations. To assess the impacts of welding deformations on the CCL positions, welding qualification trials have been performed.

Also, another factor for deformations of both a WP and a TFCC is gravitational deformations. If both deform in the similar way, we can align the datum of both and the gravitational deformation is not a problem. However, the deformation of a WP is different from a TFCC. A technique to resolve this issue has been developed.

Then, the CCL information of a WP shall be transferred to a reference points on TFCC and be tracked down through the integration process. The calculated virtual CCL positions of WP is traceable from measurements of CCL marks. A set of 4 CCL marks are attached on the inner surface of a WP at each CCL cross sections ${ }^{3)}$ (Fig. 2). Since the WP will be completely enclosed within the TFCC after Closure Welding, some techniques are 
necessary for precise evaluation of the final CCL positions.

In this paper, the descriptions of solutions for technical issues mentioned above are organized as follows:

1) Welding deformation measures in Chapter 3 ,

2) gravitational deformation measure in Chapter 4, and

3) high precision control of CCL positions in Chapter 5 .

\section{Welding Deformation Measures}

Welding qualification trials are performed for different welds of Closure Welding. The measurement results from welding qualification trials are assessed to understand the impacts on the final TF coil shape and the CCL positions.

\subsection{Closure Welding deformation}

Closure Welding is divided into 3 steps; butt welding of $\mathrm{AU}$ and BU, poloidal welding to integrate AP and BP, and Splice Plate (SP) welding (Fig. 3 and Fig. 1 of [5]). Welding deformations from those steps have different impacts on the TFCC shape and CCL positions.

\subsubsection{Butt welding}

In butt welding, 5 to $7 \mathrm{~mm}$ shrinkage across each weld line of about $100 \mathrm{~mm}$ depth is expected from welding qualification outcomes. The total extra length of $6 \mathrm{~mm}$ is given at each weld line to compensate for the welding shrinkage. The welding sequences are determined and adjusted to control BU inclination with respect to AU. Since there are $\pm 3 \mathrm{~mm}$ positional tolerances on the interface holes machined on $\mathrm{BU}$, an inclination of $\mathrm{BU}$ with respect to AU must be controlled within $\pm 3 \mathrm{~mm}$. After butt welding, WP may be tilted to align the WP plane to TFCC datum keeping the alignment at the straight portion.

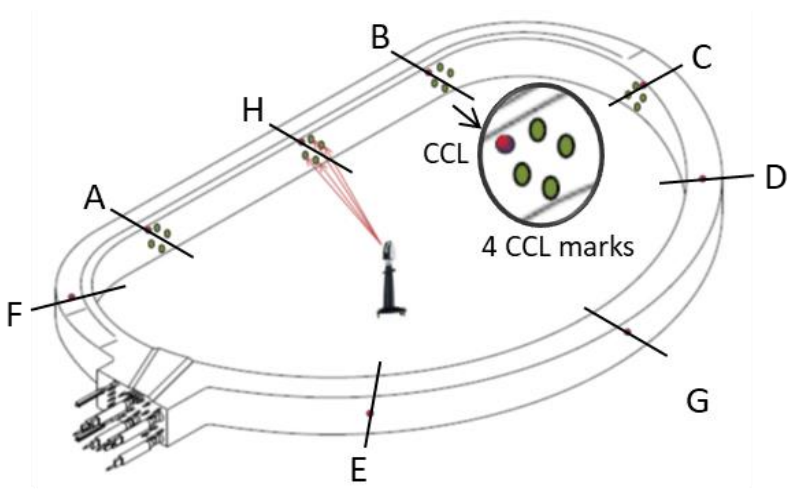

Fig. $2 \mathrm{CCL}$ cross sections, A to $\mathrm{H}$, and CCL marks. The figure shows a laser tracker at the center of a WP for CCL mark measurements. At each cross section, there are virtual CCL position indicated by red dot, and physical 4 CCL marks indicated by green dots on the inner surface of the WP to track down the CCL position.

\subsubsection{Poloidal welding}

In poloidal welding, side plates of $\mathrm{AU}$ and $\mathrm{BU}$ are expected to lean inward for 4 to $5 \mathrm{~mm}$ due to welding shrinkage. Since there is $4 \mathrm{~mm}$ minimum gap requirement on gaps between a WP and a TFCC for proper resin injection during Gap-filling operation, the side plate inclination shall be considered in determination of the WP positioning. The gap analysis is performed prior to WP Insertion to confirm the satisfaction of the gap requirement ${ }^{4)}$.

\subsubsection{SP welding}

During SP welding, the welding shrinkage over the weld lines are expected to be 2 to $3 \mathrm{~mm}$ from qualification trial results. There are $4 \mathrm{SP}$ weld lines in $31.3 \mathrm{~m}$ inner circumference of a TF coil; therefore, the shrinkage is $0.04 \%$ of the total inner length of the whole coil. Since the SP welds are located near major axis of D-shape, the deformation by welding is expected to be a small shrinkage of the distance between $\mathrm{AU}$ and BU. The qualification trial of SP welding was performed with partial model; therefore, the impact on the overall shape of a TFCC was not numerically assessed.

\subsection{Welding deformation impacts}

If the WP integration is performed in horizontal orientation, a WP needs to be supported from the side plates of $\mathrm{AU}$ and BU and the shape of the WP is impacted by the welding deformation of Closure Welding. In a concern for the impacts of much larger welding deformations than the tolerances of CCL positioning, WP integration in vertical orientation was selected in Japan. In this orientation, a TF Coil is standing on the back of straight portion $\left(90^{\circ}\right.$ rotated from the final orientation at ITER site).

In this case, the bottom surface is the only contact surface between the WP and the TFCC. Therefore, BU inclination during butt welding would not impact the shape of WP. Moreover, the WP is independent from the side plates of $\mathrm{AU}$ and $\mathrm{BU}$; therefore, the relative position

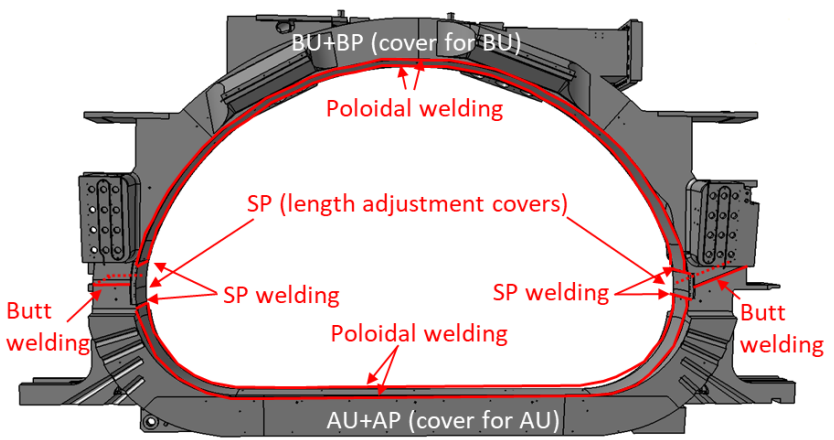

Fig. 3 Closure Welding and TFCC subassemblies. 
or shape of the WP would not change by the side plate inclination during poloidal welding.

As described previously, the shrinkage during SP welding may change the overall shape of TFCC, including the straight portion, even though the amount of the shrinkage is very small. However, the impact on the straight portion is difficult to assess directly from the amounts of welding shrinkage due to D-shape of the coil. The impact should be confirmed by measuring the WP positions and the TFCC reference points after SP welding. However, the last opportunity to measure CCL marks on the inner surface of a WP is before AP and BP installation in poloidal welding. A technique to monitor the CCL positions after butt welding was developed as described in Chapter 5.

\section{Gravitational Deformation Measure}

\subsection{Gravitational deformation analysis}

The WP fabrication is performed in horizontal orientation and therefore, the CCL positions of a WP is defined in the horizontal orientation. For a final TF coil, the CCL positions of the WP is the best CCL positions this coil can achieve without any additional stress on the WP. Therefore, the goal of the WP deformation correction is to reproduce the CCL positions of the WP when the TF coil is placed horizontal after integration.

From structural analysis, we expect that the curved region of a WP sags down for about $3.5 \mathrm{~mm}$ and the straight portion bends concave up for $1 \mathrm{~mm}$ when the orientation of the WP is changed from horizontal to vertical (Fig. 4). The analysis was repeated for a TFCC, resulting in about $1.0 \mathrm{~mm}$ and $0.2 \mathrm{~mm}$ deformations. This

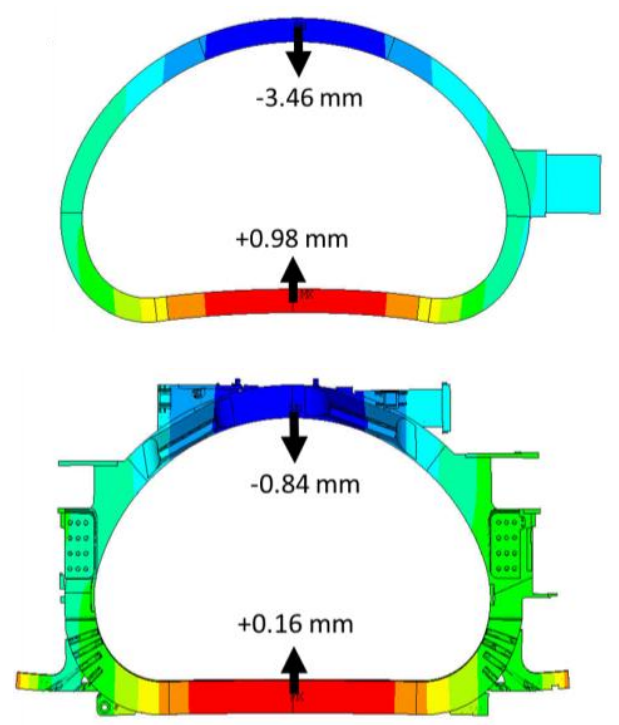

Fig. 4 Gravitational deformation of a WP (upper) and a TFCC (lower). structural analysis was performed with the boundary conditions which simulate the conditions of a WP and a TFCC in the integration process.

When each component is oriented in vertical and returned to horizontal, it will return to the original shape. Since the amount of deformations are different between a WP and a TFCC, when they are integrated together, the shape would not return to the original. The amount of return would be somewhat middle of two components.

\subsection{Correction of WP deformation}

To satisfy the $\varphi 2.6 \mathrm{~mm}$ cylindrical tolerance at the straight portion, the gravitational deformation of the WP at the straight portion must be corrected to the same level as the TFCC before finalization of WP-TFCC relationship in Gap-filling. Once their deformation is matched, the straight portion will deform back to the ideal shape when placed in horizontal orientation with proper supports.

Since a WP is completely enclosed by a TFCC after Closure Welding and the WP shape correction method shall keep the corrected shape until the relationship between the WP and the TFCC is fixed by Gap-filling, the correction of WP deformation was done by pushing the WP against the TFCC.

Sets of 7 bolts are installed at two locations at $25^{\circ}$ from the center line of curved region (Fig. 5). The amount of adjustment is controlled by the change in bolt depth while monitoring the change in the relative positions at a hole at the center. In nominal case, the target value for the relative movement at the monitoring hole is $2.9 \mathrm{~mm}$ so that the

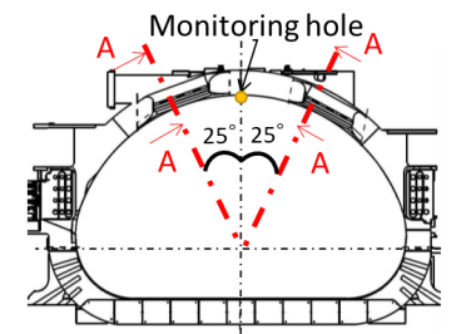

Push bolts installed at 2 locations.

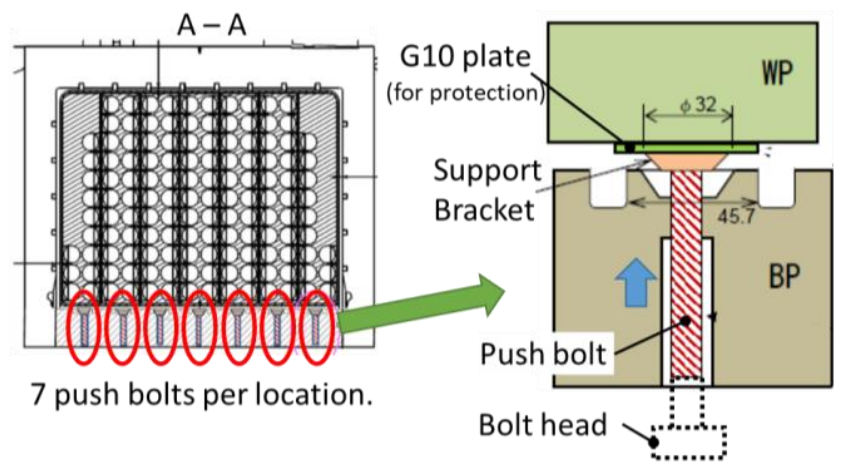

Fig. 5 WP shape correction. A WP is pushed against a TFCC by pushing bolts. 
deformation of a WP at the straight portion matches that of a TFCC. The value was calculated from the structural analysis results of displacements of a WP and a TFCC at the monitoring hole in case that the pushing forces necessary to push down the straight portion of the WP for $0.82 \mathrm{~mm}$ was applied. In the actual production, the target value needs to be calculated from the deformation amount determined from measurement results of each WP.

\section{High Precision Control of CCL Positions}

The CCL positions of a WP is transferred to TFCC reference points. However, as mentioned previously, welding deformations and gravitational deformations would cause changes in the CCL positions. Those impacts are assessed from precise measurements of WP and TFCC at every stage of manufacturing and reflected into the CCL evaluation. Developed techniques for precise control of CCL or WP positions are described below for each manufacturing step.

\subsection{WP Insertion}

During WP Insertion, a WP is positioned within a TFCC to align its CCL plane to the TFCC datum. The CCL plane is a best-fit plane of CCL positions of a WP with weights on the straight portion; constituted by a best-fit line of CCL positions at the straight portion (cross sections A, B and $\mathrm{H}$ ) as an axis and rotating the frame till the deviations in CCL positions at the curved portion (cross sections $\mathrm{C}$, D, E, F and G) are equally distributed (Fig. 2).

Since the positioning during WP Insertion has the largest impact in the final CCL positions, very precise alignment is necessary, especially at the straight portion. To align the CCL position at straight portion precisely, shims are placed between a WP and inner surface of straight portion of a TFCC at cross section A and B. By adjusting the shim thickness, vertical positions are well controlled. The CCL marks are monitored with a laser tracker against the target values in TFCC coordinate system $^{6}$. With the laser tracker, transverse positioning can be controlled under $0.3 \mathrm{~mm}$ deviations at the straight portion.

\subsection{Closure Welding}

As described previously, Closure Welding is composed of butt welding, poloidal welding, and SP welding. During those process, it is important to transfer the CCL positions of WP to the TFCC and keeping track of the information with some adjustments if necessary.

After AU-BU butt welding, the CCL marks are measured along with the TFCC reference points. By doing so, the CCL positions of the WP is related to the TFCC reference points, called Benchmark Transfer. During poloidal and SP welding, the welding deformation of the

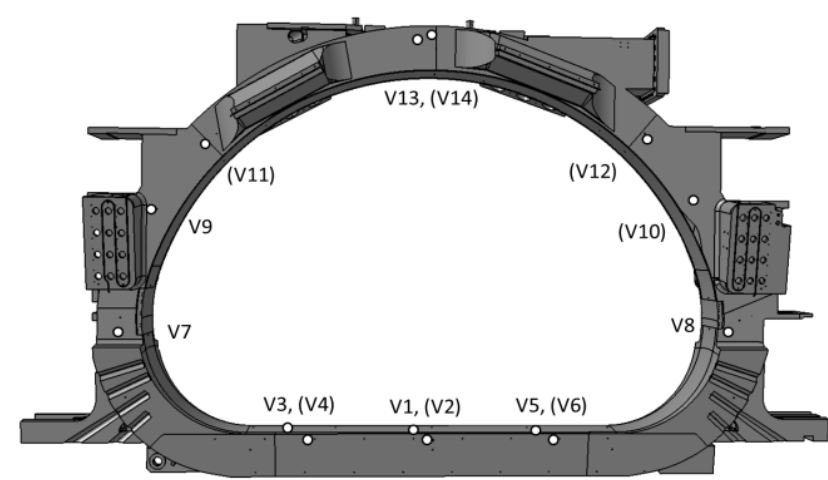

Fig. 6 Impregnation Hole Locations. Impregnation holes V1, V3, V5, V7-V9 are used for WP position measurements. Ones in parentheses are located on the opposite side.

TFCC will impact the relation between the WP and the TFCC as previously described. Therefore, the WP position and TFCC reference points shall be monitored through Closure Welding.

After poloidal welding, the WP can be only accessible through $\varphi 22 \mathrm{~mm}$ impregnation holes machined on the side surfaces of the TFCC for the resin injection during Gapfilling (Fig. 6). WP position plates are attached on a side of WP at the locations of impregnation holes. Those plates are measured with laser tracker before and after Closure Welding so that the change in WP condition is traceable. However, the locations of those impregnation holes are selected for proper Gap-filling and do not necessarily coincide with the CCL cross sections.

The change in the CCL positions need to be calculated from the change in WP position plates measurements. The evaluation utilizes mechanical simulation ${ }^{7)}$ inputting displacements of WP position plates as imposed displacements to calculate the corresponding displacements at the CCL positions of WP. The WP model in structural analysis is prepared considering the material compositions and their properties. Those models have been verified and updated through comparison to the actual measurement results of the gravitational deformation of a WP.

The outputted displacements at the CCL positions due to Closure Welding, $\delta \boldsymbol{x}_{C W}$, are added to the CCL positions at the Benchmark Transfer, $\boldsymbol{x}_{C C L}$, to determine the CCL positions after Closure Welding, $\boldsymbol{x}^{\text {' }}{ }_{C L}$.

$$
\boldsymbol{x}_{C C L}^{\prime}=\boldsymbol{x}_{C C L}+\delta \boldsymbol{x}_{C W}
$$

Thus, the tracking technique for CCL positions are developed in order to evaluate CCL position as precise as the required high accuracy.

\subsection{Gap-filling}

The above technique is utilized for the tracking of the 
CCL positions through WP shape correction as well. Then, the CCL positions after WP shape correction, $\boldsymbol{x}$ " $C C L$ is expressed with the displacements in the CCL positions due to WP shape correction, $\delta \boldsymbol{x}_{W S C}$ as Eq. (2).

$$
\boldsymbol{x}_{C C L}^{\prime \prime}=\boldsymbol{x}_{C C L}+\delta \boldsymbol{x}_{C W}+\delta \boldsymbol{x}_{W S C}
$$

The reference points on the TFCC are also measured in the same coordinate system to finalize the Benchmark Transfer.

During Gap-filling, a WP position is monitored and controlled so that the relationship between the WP and the TFCC would not change. The factors for deviations in the relative position are; 1) the temperature difference between the WP and the TFCC and 2) internal and external pressure difference at the terminal region of the $\mathrm{WP}^{8)}$. The temperature difference and the pressure difference were controlled while monitoring the relative position of WP at the terminal region using micrometers. Then, they are physically fixed by the gap-filling resin after cure. The CCL positions are finalized within the TFCC datum and related to the reference points on the TFCC surface.

\subsection{Final Machining}

At Final Machining, the machining axis is determined considering the CCL deviations and the extra materials on the interface surfaces of the TFCC. The TFCC datum is shifted or rotated if necessary, to minimize the CCL deviations. As described previously, there is a severe tolerance of $\pm 0.2 \mathrm{~mm}$ at the side surfaces of the straight portion. This requirement was achievable by use of high precision milling machine and the precise alignment of the TF coil on the milling machine.

\section{Application to TF Coil Production}

The developed techniques are applied to the TF coil production. Manufacturing of two TF coils has been completed so far. Detail descriptions of the CCL evaluation for the first coil is reported. Since the CCL evaluation for a TF coil is not a simple measurement but involves a series of calculations throughout the WP integration process, uncertainties in evaluation cannot be ignored. The uncertainties are also calculated to confirm the satisfaction of CCL tolerances regardless of the uncertainties.

\subsection{WP Insertion}

In WP Insertion, WPs deformed by gravitation by 0.81 $\mathrm{mm}$ upward at the straight portion and $3.07 \mathrm{~mm}$ downward at the curved region in vertical direction, similarly to the mechanical simulation predictions. There were maximum of $0.28 \mathrm{~mm}$ out-of-plane deformations appeared in the straight portion. The weight of the WP might have suppressed the deformation during WP assembly. As previously described, such unexpected deviations are compensated during Final Machining.

Regardless of the deformation, alignments of the CCL positions are controlled within $0.31 \mathrm{~mm}$ of target values for the first coil. The outcomes for the following coils are summarized in Table 1. Out-of-plane deformations and alignment deviations are only assessed for the straight portion since the alignment at the curved portion is adjusted after butt welding. The outcomes show the establishment of precise and stable alignment control in WP Insertion.

\subsection{Closure Welding}

During Closure Welding, the amounts of welding deformations were assessed; 6 to $7.5 \mathrm{~mm}$ shrinkage over the welds and $2.2 \mathrm{~mm}$ BU inclinations by butt welding, and maximum of $5 \mathrm{~mm}$ side plate inclinations during poloidal welding for the first coil. The results of Closure Welding deformation are summarized in Table 2. The amounts of the deformation conform with the expected values assessed from welding qualification trials as presented in Chapter $3 ; 5$ to $7 \mathrm{~mm}$ shrinkage over butt welding and 4 to $5 \mathrm{~mm}$ side plate inclination due to poloidal welding.

To align the CCL plane of the WP and the TFCC reference frame, the WP tilt is adjusted after butt welding. Then, wedged shims are inserted at the cross-section G of Fig. 2 to keep the adjusted position.

Table 1 Deformation and deviations in WP Insertion. Upward direction is indicated positive in vertical deformation. All in $\mathrm{mm}$.

\begin{tabular}{|c|l|c|c|c|c|}
\hline \multicolumn{2}{|c|}{ Coil } & $1^{\text {st }}$ & $2^{\text {nd }}$ & $3^{\text {rd }}$ & $4^{\text {th }}$ \\
\hline $\begin{array}{c}\text { Vertical } \\
\text { deformation }\end{array}$ & $\begin{array}{l}\text { Straight } \\
\text { portion }\end{array}$ & +0.81 & +0.92 & +0.88 & +0.90 \\
\cline { 2 - 6 } & $\begin{array}{l}\text { Curved } \\
\text { portion }\end{array}$ & -3.07 & -2.70 & -3.26 & -3.13 \\
\hline $\begin{array}{c}\text { Max. absolute out-of- } \\
\text { plane deformation }\end{array}$ & 0.28 & 0.07 & 0.55 & 0.27 \\
\hline $\begin{array}{c}\text { Max. absolute } \\
\text { alignment deviation }\end{array}$ & 0.31 & 0.39 & 0.27 & 0.38 \\
\hline
\end{tabular}

Table 2 Closure Welding deformation. All in $\mathrm{mm}$.

\begin{tabular}{|c|c|c|c|c|c|}
\hline Coil & & $1^{\text {st }}$ & $2^{\text {nd }}$ & $3^{\text {rd }}$ & $4^{\text {th }}$ \\
\hline \multirow{2}{*}{$\begin{array}{c}\text { Shrinkage over } \\
\text { butt welds }\end{array}$} & Min & 6.0 & 6.0 & 6.0 & 4.5 \\
\cline { 2 - 6 } & Max & 7.3 & 7.5 & 7.5 & 7.0 \\
\hline \multicolumn{2}{|c|}{ Absolute BU Inclination } & 2.2 & 1.2 & 0.2 & 2.3 \\
\hline \multirow{2}{*}{$\begin{array}{c}\text { Max. side plate } \\
\text { inclination }\end{array}$} & $\begin{array}{c}\text { Straight } \\
\text { portion }\end{array}$ & 4.5 & 4.6 & 4.7 & 4.9 \\
\cline { 2 - 6 } & $\begin{array}{c}\text { Curved } \\
\text { portion }\end{array}$ & 5.0 & 4.8 & 4.6 & 5.2 \\
\hline
\end{tabular}




\subsection{Gap-filling}

Before Gap-filling, WP shape was corrected as planned. The correction amount was determined from the WP deformation for each coil reported in Table 1. The correction targets and results are summarized in Table 3. Then, the WP position plates and the TFCC refence points are measured with laser tracker to evaluate the CCL positions.

During Gap-filling, the relative positions of WPs to TFCCs are controlled to maintain the relationships. As a result, only $0.2 \mathrm{~mm}$ relative displacements in longitudinal direction are observed and less than $0.1 \mathrm{~mm}$ in transverse direction for the first coil at the terminal region ${ }^{8)}$. The impact of the relative displacement on CCL positions are negligible. The finalization of the relationship between the WP and the TFCC was completed.

\subsection{Final Machining}

Even though WP positioning was well controlled during WP Insertion, due to welding deformations during Closure Welding, the deviations in CCL positions are increased. In order to satisfy the tolerance requirements defined for the final TF coil, the CCL positions need to be optimized by adjusting machining axis to compensate for the deviations.

Final Machining is performed in horizontal orientation, the same orientation and the same support positions as Factory Acceptance Tests. During assembly at the ITER site, TF coils are stood with the straight portion vertical;

Table 3 WP shape correction results. All in $\mathrm{mm}$. Monitoring hole location is shown in Fig. 5.

\begin{tabular}{|c|c|c|c|c|c|}
\hline Coil & & $1^{\text {st }}$ & $2^{\text {nd }}$ & $3^{\text {rd }}$ & $4^{\text {th }}$ \\
\hline $\begin{array}{c}\text { Relative } \\
\text { displacement at } \\
\text { monitoring hole }\end{array}$ & Target & 2.40 & 2.48 & 2.83 & 3.08 \\
\cline { 2 - 6 } & Result & 3.00 & 2.52 & 2.80 & 3.10 \\
\hline $\begin{array}{c}\text { CCL correction at } \\
\text { section H }\end{array}$ & Target & 0.68 & 0.63 & 0.81 & 0.88 \\
\cline { 2 - 6 } & Result & 0.87 & 0.84 & 0.58 & 0.79 \\
\hline
\end{tabular}

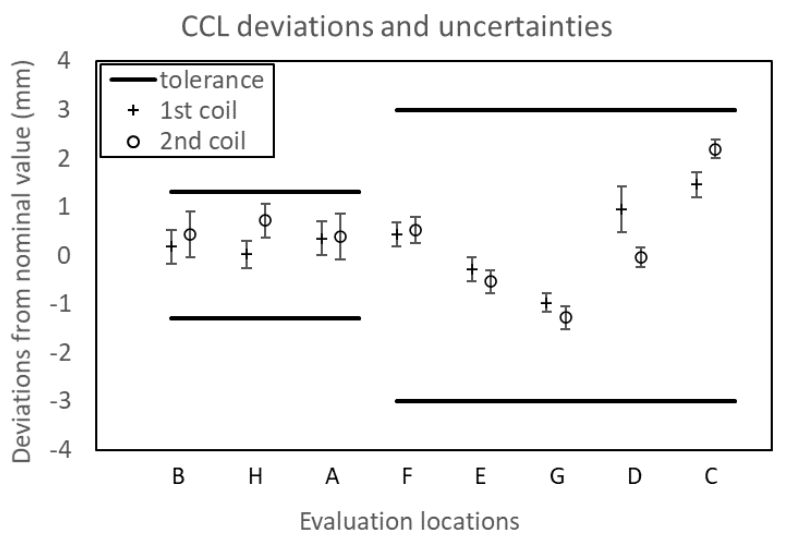

Fig. 7 Deviations and uncertainties in CCL positions of the TF coils. however, each coil is supported against the gravitational deformations. By properly supporting TF coils in horizontal orientation, it was agreed to evaluated the final CCL positions in horizontal orientation during Factory Acceptance Tests.

For the first coil, the machining axis was shifted 0.5 $\mathrm{mm}$ to compensate for the CCL deviations. By doing so, the deviations are optimized to be within $\varphi 0.5 \mathrm{~mm}$ from the nominal at the straight portion. No adjustment was necessary for the second coil.

\subsection{Final CCL positions and their uncertainties}

The deviations in CCL positions from nominal values are shown in Fig. 7. The horizontal axis is the positions of CCL cross section counted counter-clockwise starting from cross section B. In the vertical axis, the deviation at each cross section is given as cross point. The lengths of lines extended from the point indicate the uncertainties in the CCL position evaluated.

The uncertainties in the final CCL evaluation results are calculated from the uncertainties in the steps of WP integration. Also, the uncertainties in CCL positions of a WP propagates through the WP integration process to final CCL positions of TF coil. The maximum values among all the cross sections for each coil are summarized in Table 4.

a) Uncertainties in CCL positions of a WP,

b) Measurement uncertainty during Benchmark Transfer,

c) Impact of measurement uncertainty during WP deformation correction on CCL coordinate calculation, and

d) Impact of measurement uncertainty during Factory Acceptance Test on CCL coordinate calculation.

In Benchmark Transfer, CCL marks and TFCC reference points are measured with laser tracker. The measurements are repeated for several times per measurement point to minimize measurement errors. The CCL positions are calculated using the vector relations as

Table 4 Uncertainty contribution of each step to CCL positions of the final coil. All in mm. N/A indicates no data available yet.

\begin{tabular}{|c|c|c|c|c|}
\hline Coil & $1^{\text {st }}$ & $2^{\text {nd }}$ & $3^{\text {rd }}$ & $4^{\text {th }}$ \\
\hline $\begin{array}{c}\text { Max. contribution from WP } \\
\text { fabrication }\end{array}$ & 0.27 & 0.20 & 0.22 & 0.22 \\
\hline $\begin{array}{c}\text { Max. contribution from } \\
\text { Benchmark Transfer }\end{array}$ & 0.14 & 0.02 & 0.03 & 0.02 \\
\hline $\begin{array}{c}\text { Max. contribution from WP } \\
\text { shape correction }\end{array}$ & 0.05 & 0.23 & 0.09 & 0.08 \\
\hline $\begin{array}{c}\text { Max. contribution from Factory } \\
\text { Acceptance Test }\end{array}$ & 0.07 & 0.16 & $\mathrm{~N} / \mathrm{A}$ & $\mathrm{N} / \mathrm{A}$ \\
\hline
\end{tabular}


described in [3], the mean of 4 vectors, $\bar{x}$, is adopted as the resultant value. Standard deviation of the mean is defined as Eq. (3).

$$
\sigma_{\bar{x}}=\frac{\sigma_{x}}{\sqrt{4}}
$$

$\sigma_{\bar{x}}$ indicates standard deviation of the mean, while $\sigma_{x}$ is the standard deviation of the 4 vectors. Following this definition, the measurement uncertainties of CCL marks are evaluated to be $0.14 \mathrm{~mm}$ maximum for the first coil assuming the normal distribution of the measurement data.

During WP shape correction, the measurements of the WP position plates and the TFCC reference points are also repeated to optimize measurement uncertainties. Those measurement uncertainties are used as input for structural analysis to assess the impact on the CCL evaluation.

The uncertainty contributions for CCL positions summarized in Table 4 show that even though the measurements are taken in factory conditions with vibration and dust from surrounding machines, the repetition of the measurements decreased the measurement uncertainties to the acceptable level around $0.2 \mathrm{~mm}$.

Figure 7 shows that the total values of deviations and uncertainties are within the tolerance requirements of $\varphi 2.6 \mathrm{~mm}$ at the straight portion and $\pm 3 \mathrm{~mm}$ at the curved portion were satisfied for the first two TF coils. The CCL evaluation techniques for final TF coil is deemed adequate for application in TF coil production.

\section{Conclusion}

To satisfy severe requirements given for magnetic property of ITER TF coils, WP integration is performed in vertical orientation with precise control and adjustment of WP position and correction of the WP shape. Because the CCL characterized the magnetic property of a coil, the CCL positions of a TF coil are evaluated from the laser tracker measurement results obtained in different steps of WP integration. With those control and evaluation techniques, the impact of welding deformations, gravitational deformation and deviation during Gapfilling were minimized in the manufacturing process. As a result, two TF coils have been fabricated successfully, satisfying the ITER requirements. Currently, four TF coils are in WP integration phase. The WP integration process is in series production phase in Japan.

\section{Acknowledgments}

The author would like to thank Mitsubishi Heavy Industries, Ltd and Mitsubishi Electric Corp. for their contribution in the manufacturing process qualification and implementation to TF coil manufacturing. We have faced many issues and troubles during the planning and manufacturing of the first TF coil and it was their sincere passion towards craftsmanship that encouraged us to move this project forward. It was our pleasure to work with them.

\section{References}

1) J. Kanster and E. Baynham: "The determination of the current center line of the TF coils of ITER," IEEE Trans. Appl. Supercond. 20 (2010) 1475-1478

2) J. Knaster, et al.: "Final assembly and installation of the ITER TF coils," IEEE Trans. Appl. Supercond. 18 (2008) 495-499

3) H. Kajitani, et al.: "Development of ITER toroidal field coil winding packs - Results of winding pack development -," TEION KOGAKU 55 (2020) 338-345 (in Japanese)

4) N. Koizumi, et al: "Development of ITER TF coil winding pack (WP) and qualification for assembling WP and coil case in Japan," IEEE Trans. Appl. Supercond. 29 (2019) 4200505

5) M. Nakahira and N. Koizumi: "Manufacturing of ITER toroidal field coil in Japan - TF coil structure and integration -," TEION KOGAKU 55 (2020) 381-384 (in Japanese)

6) E. Baynham, et al.: "The insertion of the WP in the structural casing of the TF coils of ITER," IEEE Trans. Appl. Supercond. 20 (2010) 389-393

7) N. Koizumi, et al.: "Progress of ITER TF coil fabrication in Japan," IEEE Trans. Appl. Supercond. 30 (2020) 4202106

8) M. Nakamoto, et al.: "Development of gap-filling impregnation method of ITER TF coils", TEION KOGAKU 55 (2020) 409-417

Mio NAKAMOTO Mio Nakamoto holds a Bachelor in Scienece in Physics from University of California, Los Angeles. She joined National Institues for Quantum and Radiological Science and Technology (QST) in 2015 as a temporary staff. In 2018, she became the full-time staff and appointed as the responsible person for WP integration of TF coils.

Yuma KASAI Yuma Kasai graduated from Tohoku University and joined Mitsubishi Electric Corporation in 2013. He served for manufacturing of ITER TF coil and superconducting device. Currently, he works at QST as a Part-time Researcher.

Takashi BABA Takashi Baba joined QST in 2019 as a temporary staff. $\mathrm{He}$ is in part of the integrating of the TF coil and its accessories.

Kazuyuki SAKAMOTO Kazuyuki Sakamoto joined QST in May 2017. $\mathrm{He}$ is in part of integrating TF coils and transport frames for TF coils.

Tatsuya SHIMIZU Tatsuya Shimizu jointed QST in Nov. 2000 as a structural analysis specialist. He is in part of the structure analysis of the TF coil.

Kengo SAITO Kengo Saito joined QST in April 2017 as a CAD specialist. He is in part of the evaluation of measrument of the TF coil. 
Norikiyo KOIZUMI Born in May 1964. He graduated from Waseda

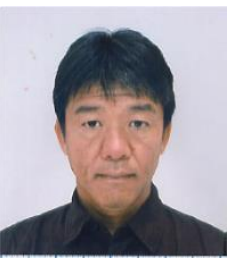
University and joined Japan Atomic Energy Agency (JAEA) in 1990. He has been engaged in research and development of superconductors and magnets for nuclear fusion reactor use. He holds a $\mathrm{Ph} . \mathrm{D}$ in Engineering.

Masataka NAKAHIRA Born in March 1967. Holding a Doctor of

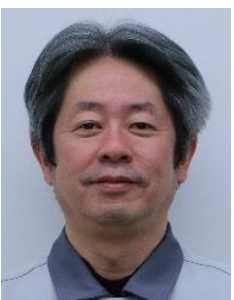

Engineering from Tsukuba University, received in 2004. He joined the superconducting magnet technology group in JAEA / QST from 2013, after 5 years employment at ITER, France. He works as a group leader of the superconducting magnet technology group since 2019 . He worked for ITER since 1992 starting from JAERI.

Eikou FUJIWARA Born in May 1982. He graduated from Ritsumeikan University and joined Mitsubishi Heavy Industries in 2008. He served for manufacturing of ITER TF coil case when he worked at QST from 2016 to 2018 as a Part time Reasercher. Currentlty, he works at Mitsubishi Heavy Industries and served for assembling TF coil case and coil as project engineer.
Minoru YAMANE Minoru Yamane joined Mitsubishi Electric Corporation in 1985 and has been engaged in the design of Nuclear Fusion experimental drvices.

Tsuneaki MINATO Tsuneaki Minato holds a Doctor in Engineering in Osaka University, Japan. He joined Mitsubishi Electric Corporation in 1983 and has been engaged in the design of magnetic coils of Nuclear Fusion experimental devices. He engages in the production design of the ITER TF coil from 2012.

Kazuo KUNO Master's degree holder from Kyoto University. Jointed Mitsubishi Electric Corporation in 1974. Have been working in the field of superconducting magnet, fusion devices. Now project leader in Mitsubishi Electric for ITER TF Coil. 